\title{
Building Magnetoresponsive Composite Elastomers for Bionic Locomotion Applications
}

\author{
Yi Lei ${ }^{1}$, Zhizhi Sheng ${ }^{2,3}$, Jian Zhang ${ }^{2}$, Jing Liu ${ }^{2}$, Wei Lv ${ }^{1}$, Xu Hou ${ }^{1,2,3^{*}}$ \\ 1. Department of Physics, Research Institute for Biomimetics and Soft Matter, Jiujiang Research Institute, College of Physical Science \\ and Technology, Xiamen University, Xiamen 361005, China \\ 2. State Key Laboratory of Physical Chemistry of Solid Surfaces, College of Chemistry and Chemical Engineering, Xiamen University, \\ Xiamen 361005, China \\ 3. Collaborative Innovation Center of Chemistry for Energy Materials, Xiamen University, Xiamen 361005, China
}

\begin{abstract}
The ability of natural living organisms, transferring deformations into locomotion, has attracted researchers' increasing attention in building bionic actuators and smart systems. As a typical category of functional materials, magnetoresponsive composite elastomers, comprised of flexible elastomer matrices and rigid magnetic particles, have been playing critical roles in this field of research due to their dynamic changes in response to applied magnetic field direction and intensity. The magnetically driven bionic actuators based on magnetoresponsive composite elastomers have been developed to achieve some specific functions in some special fields. For instance, under the control of the applied magnetic field, the bionic actuators can not only generate time-varying deformation, but also motion in diverse environments, suggesting new possibilities for target gripping and directional transporting especially in the field of artificial soft robots and biological engineering. Therefore, this review comprehensively introduces the component, fabrication, and bionic locomotion application of magnetoresponsive composite elastomers. Moreover, existing challenges and future perspectives are further discussed.
\end{abstract}

Keywords: magnetoresponsive composite elastomers, components, fabrication, bionic locomotion

Copyright $(C)$ The author(s) 2020.

\section{Introduction}

The excellent shape-shifting abilities and capabilities of transforming deformations into diverse motions to adapt themselves to complex survival environments of some creatures, such as caterpillars ${ }^{[1]}$, inchworms ${ }^{[2]}$, jellyfishes ${ }^{[3]}$, nematodes ${ }^{[4]}$, snakes ${ }^{[5]}$, octopus ${ }^{[6]}$, have inspired scientists to devote themselves to designing bionic actuators that can deform and motion in response to an external stimulus (Fig. 1). The bionic actuators based on magnetoresponsive composite elastomers have attracted increasing attention due to the excellent deformability and magnetic responsiveness of magnetoresponsive composite elastomers. The magnetoresponsive bionic actuators can clamp a target, overcome obstacles and finally deliver them to destination ${ }^{[7,8]}$, providing new possibilities to develop smart devices in the promising fields of biomedicine ${ }^{[9-13]}$, artificial soft robotics $^{[14-16]}$, microfluidic systems ${ }^{[17,18]}$, flexible electronics $^{[19]}$, etc.
Generally, magnetoresponsive composite elastomers for bionic locomotion applications are mainly composed of flexible elastomeric matrices like natural rubber $^{[20-22]}$, silicone rubber ${ }^{[23-25]}$, polyurethane rubber ${ }^{[26-29]}$, polybutadiene rubber ${ }^{[30]}$, and embedded hard micro-sized or nano-sized magnetic particles. To improve their mechanical performance or endow them with exceptional characteristics, a small quantity of additives is admixed. Depending on the structure of embedded magnetic particles, magnetoresponsive composite elastomers can be classified into isotropic materials where magnetic particles are uniformly dispersed in matrices and anisotropic materials where magnetic particles form special chain-like or pillar-like structure in matrices.

Magnetoresponsive composite elastomers are constructed by simply mixing ingredients, and then curing the mixture at an appropriate temperature. Magnetoresponsive films with adjustable thickness can be obtained by spin or spray coating the uncured mixture. Coupling with the magnetic particle-assisted molding

\footnotetext{
*Corresponding author: $\mathrm{Xu} \mathrm{Hou}$

E-mail: houx@xmu.edu.cn
} 

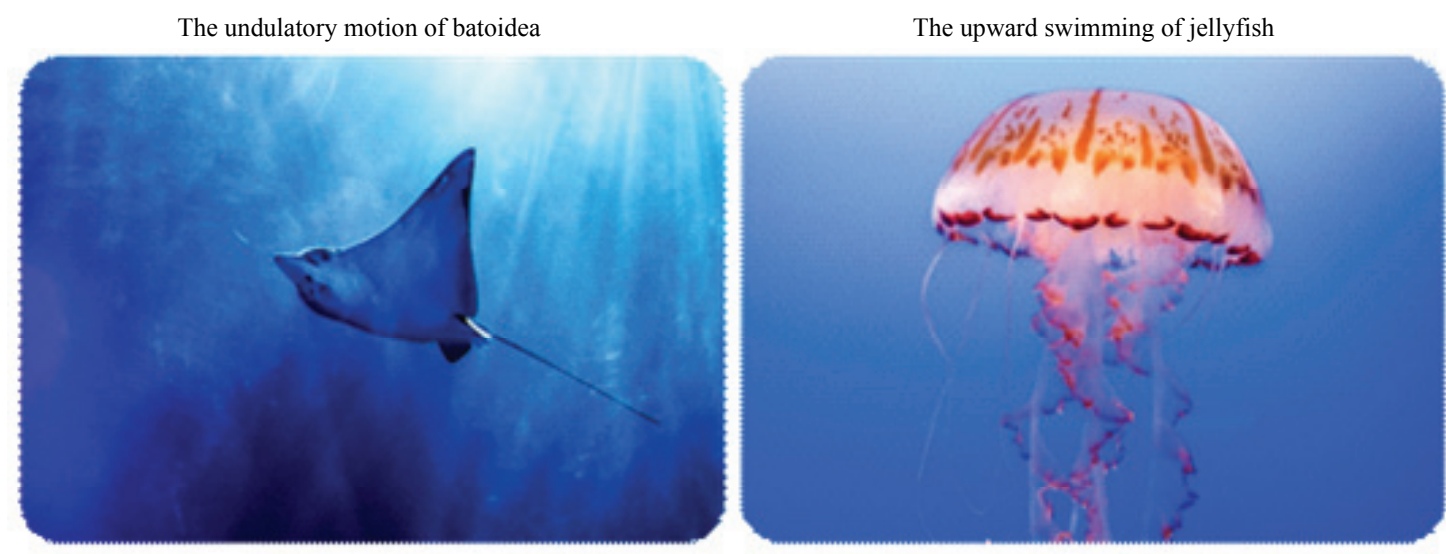

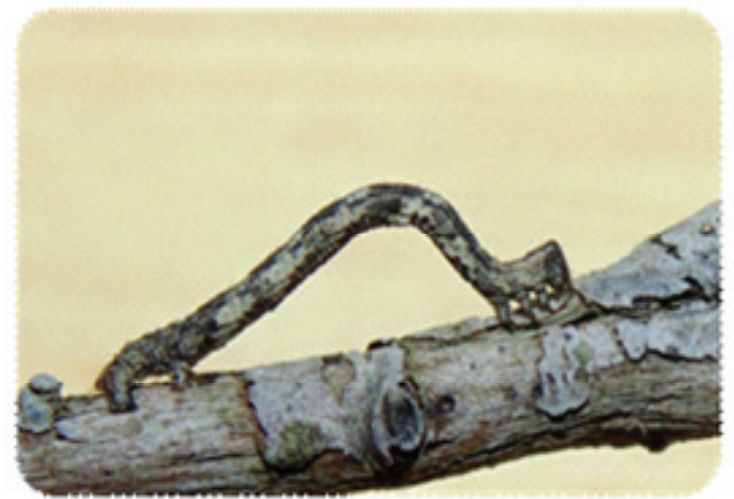

The telescoping motion of inchworm

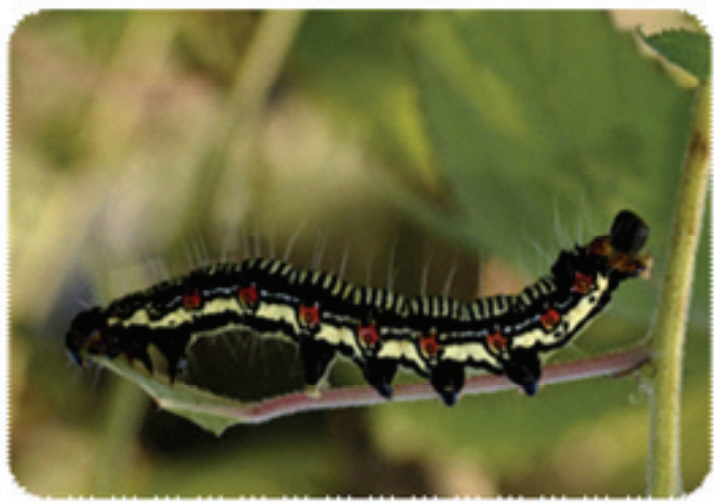

The crawling locomotion of caterpillar

Fig. 1 The shape-morphing movements of batoidea, jellyfish, inchworm, caterpillar, and other living organisms provide endless inspiration for scientists to build bionic actuators based on magnetoresponsive composite elastomers. These magnetoresponsive bionic actuators can deform and move at various environments like creatures with the action of a magnetic field, which have potentials to be applied as smart devices in the fields of artificial soft robotics, biomedicine, microfluidic systems, and flexible electronics. The photographs of batoidea, inchworm, and caterpillar are downloaded from the website of Pixabay (https://pixabay.com/zh/), and the photograph of jellyfish is downloaded from the website of Unsplash (https://unsplash.com/).

process, three-dimensional motile magnetoresponsive pillars are formed. However, to satisfy their broad scope of applications, magnetoresponsive composite elastomers with various geometrics are required, provoking the development of more intelligent manufacturing technologies ${ }^{[31-33]}$. One of the currently available approaches is to adopt the three-dimensional (3D) printing technique whose essence is the successive stacking of layers of the prepared raw materials by computer software ${ }^{[34]}$.

This review comprises of five sections. The first section gives a brief introduction of magnetoresponsive composite elastomers and their bionic locomotion applications. Then, the components of magnetoresponsive composite elastomers are overviewed in the second section, including their elastomer matrices, magnetic particles, and additives. The fabrication methods of magnetoresponsive composite elastomers containing spin coating, spray coating, and 3D printing technology are overviewed in the third section. After that, the bionic locomotion application of magnetoresponsive composite elastomers is presented in the fourth section. Finally, some existing challenges and future perspectives about the development of magnetoresponsive composite elastomers are further discussed in the fifth section.

\section{Components of magnetoresponsive compo- site elastomers}

Magnetoresponsive composite elastomers as smart materials have attracted extensive attentions due to their rapid response to the magnetic field stimulus. Their properties can be reversibly controlled by regulating the applied magnetic field parameters, especially the macroscopic shape distortion. In particular, the magnetoresponsive composite elastomers can be stretched, 
warped or recoiled easily when exposing to a magnetic field. Generally, magnetoresponsive composite elastomers are constructed by dispersing micro-sized or nano-sized magnetic hard particles into elastomeric soft matrices. However, a small quantity of additives is sometimes used to improve their performance or endow them with exceptional characteristics.

\subsection{Elastomer matrices}

Numerous polymers can be used as elastomer matrices. Among them, natural rubber ${ }^{[20-22]}$, silicone rubber $^{[23-25]}$ and polyurethane ${ }^{[26-29]}$ are three kinds of usually used matrices as we have known.

Natural rubber is the natural polymeric compound based on polyisoprene which needs to be vulcanized before use. The crosslinking process of natural rubber with the assistance of crosslinking agents, accelerators, and activators is the vulcanization, which determines the mechanical properties of natural rubber. The modulus and the elongation at break of the natural rubber with higher crosslink density are higher than those of the natural rubber with lower crosslink density, on the contrary, the tensile strength of the natural rubber with higher crosslink density is lower than that of the natural rubber with lower crosslink density. Due to its better mechanical characteristics and heat resistance than silicone rubber, natural rubber is suitable for practical engineering applications. Nevertheless, its complex vulcanization process, low reversibility, and poor aging limit the utilization of natural rubber. Chen et al. ${ }^{[20]}$ fabricated natural rubber-based magnetoresponsive composite elastomers and found that their tensile and tear strengths were far higher than that of silicone rubber-based materials. Furthermore, based on the natural rubber, a new type of biodegradable epoxidized natural rubber with better thermal resistance could be synthesized via a usual epoxidation process ${ }^{[22]}$.

Compared with the natural rubber, the poor mechanical properties and low durability of silicone rubber confine the engineering applications of silicone rubber-based magnetoresponsive composite, yet the silicone rubber is also popular due to its simple preparation process and chemicals, fungi resistances ${ }^{[35]}$. The silicone rubber precursor is liquid with low viscosity at room temperature, the magnetic particles are liable to disperse and move in the precursor, which makes it easy for the magnetoresponsive composite elastomers to form chain-like or pillar-like structures. In addition, the mechanical properties of the silicone rubber are closely correlated with the degree of curing.

Polyurethane rubber has attracted widespread attention owing to its high elasticity, better wear resistance, tear resistance, and other advantages over natural rubber and silicone rubber. Ju et al. ${ }^{[26]}$ prepared polyurethane rubber-based magnetoresponsive composite elastomers and found that the loss factor was higher than those composed of natural rubber or silicone rubber. Lian et $a l .{ }^{[27]}$ compared polyurethane-based samples to silicone-based samples and results revealed that the former showed better friction and wear performance, while the latter exhibited poor mechanical characteristics.

In addition to the abovementioned elastomer matrices, some other popular materials are utilized in actual applications, for example, isobutylene-isoprene rubber $^{[36]}$, bromobutyl rubber ${ }^{[37]}$, and mixed matrices ${ }^{[38-40]}$. Zhang et al. ${ }^{[38]}$ admixed cis-polybutadiene rubber and natural rubber. While Song et al. ${ }^{[40]}$ blended ethylene-propylene-diene monomer and methylvinyl silicone rubber. Wang et al. ${ }^{[39]}$ produced a new kind of matrix by means of reacting polychloroprene rubber with epoxidized natural rubber.

\subsection{Magnetic particles}

The variables of embedded particles include the type, shape, size, content, and coating that have prominent influences on magnetoresponsive composite elastomers. Carbonyl iron ${ }^{[41-43]}, \mathrm{Fe}_{2} \mathrm{O}_{3}{ }^{[44]}, \mathrm{Fe}_{3} \mathrm{O}_{4}{ }^{[45-47]}, \mathrm{Fe}-$ $\mathrm{Co}_{3}{ }^{[48]}, \mathrm{CoFe}_{2} \mathrm{O}_{4}{ }^{[49]}, \mathrm{Fe}^{[50,51]}$, nickel ${ }^{[52]}$, have been utilized as magnetizable particles. The high permeability, low remanence, and high saturation magnetization lead to the widespread employ of carbonyl iron particles. Hapipi et al ${ }^{[53]}$ prepared two different samples that were based on plate-like carbonyl iron particles and spherical-like carbonyl iron particles respectively. Kwon et $a l{ }^{[44]}$ impregnated needle-typed gamma iron oxide nanoparticles and carbonyl iron in natural rubber to fabricate magnetic elastomers.

As is well-known, there is a clear dependence between particle size fraction and magnetic properties. Sorokin et al. ${ }^{[54]}$ firstly investigated the effect of mag- 
netic particle size on magnetorheological response and observed that the magnetorheological response improved as an increasing fraction of large particles due to the enhanced magnetic interactions. Similarly, Winger $e t$ $a l .{ }^{[55]}$ compared the magnetorheological response of four different specimens involved in four different iron particle size fractions. With the increasing particle size, the interaction energy would increase which led to the improvement of magnetorheological response but weakened mechanical performance.

The covalent bonding between hydroxyl groups on the particle surface and silane groups of cross-like agent restricts the mobility of magnetic particles. To solve this problem, researchers come up with some modifications toward magnetic particles ${ }^{[56-60]}$. Cvek et al. ${ }^{[56-58]}$ modified carbonyl iron particles by grafting with poly (glycidyl methacrylate) (PGMA) via surface-initiated atom transfer radical polymerization, which eliminated the drawback of poor particle dispersibility, and found negligibly decreased magnetic properties but significantly enhanced thermo-oxidation stability. Later, they attempted to graft carbonyl iron particles with poly (trimethysilyloxyethyl methacrylate) (PHEMATMS) via the same method, both the magnetic and mechanical characteristics were improved.

\subsection{Additives}

Elastomer matrices and magnetic particles are the main components of magnetoresponsive composite elastomers. However, to broaden their applications, some researchers utilize additives, including plasticizers $^{[61-63]}$, reinforcing agents ${ }^{[64-66]}$ and some phase change materials. In general, small amounts of additives can contribute to improving the mechanical and magnetic properties of magnetoresponsive composite elastomers in different ways.

Plasticizers soften the elastomer matrices and reduce the viscosity of elastomers, improving the mobility of magnetic particles. In comparison with magnetoresponsive composite elastomers without any plasticizers, specimens containing phthalate esters ${ }^{[63]}$ such as butyl benzyl phthalate (BBP), dioctyl phthalate (DOP), dimethyl phthalate (DMP), bis (2-miethyloctyl) phthalate (DINP) or natural esters ${ }^{[61]}$ such as sucrose acetate isobutyrate (SAIB) ester exhibited higher magnetorheo- logical effect, enhanced storage and loss moduli. Similarly, taking silicone oil as an additive could improve the magnetic effect ${ }^{[62]}$.

Reinforcing agents strengthen the matrix, thus providing superior bonding between elastomer matrices and magnetic particles, which leads to the improved performance of magnetoresponsive elastomers. Carbon-type nanofillers are frequently used because of their lightweight and high specific surface area. Carbon black $^{[64]}$ and carbon nanotube ${ }^{[65]}$ have been validated to improve the mechanical properties of magnetoresponsive composite elastomers. Furthermore, $\mathrm{Fan}^{[67]}$ and $\mathrm{Lu}^{[68]}$ respectively investigated the effect of carbon black size and content on properties of magnetoresponsive composite elastomers. Aziz et al. ${ }^{[69,70]}$ found that functional carboxylated multiwall carbon nanotubes had better compatibility with magnetic particles.

Unlike plasticizers and reinforcing agents, the application of phase change materials influences the intelligent control. Gong et al. ${ }^{[71]}$ added polycaprolactone (PCL) which could translate into the liquid state from semicrystalline solid when the surrounding temperature was above the PCL melting point. Furthermore, Guo et $a l .{ }^{[72]}$ creatively proposed a new type of composite material comprising liquid metal and EGaIn, which endows magnetoresponsive elastomers a positive piezoconductive effect that their resistivity was at a maximal value in the initial free state and declined sharply upon any kind of mechanical deformations.

\section{Fabrication methods of magnetoresponsive composite elastomers}

Conventionally, the preparation of magnetoresponsive composite elastomers includes mixing and curing two steps. While the manufacture of anisotropic samples needs an additional magnetic field-assisted process that an external magnetic field is applied before or during curing the mixture of elastomers, magnetic particles, and additives so that the magnetic particles are orderly arranged in chain-like structures or pillar-like structures along the direction of a magnetic field. The spin and spray coating processes as simple fabrication methods, no template is needed, only a spin coater or an airbrush, are usually utilized when the thin magnetoresponsive films are needed. Combing with the magnetic 


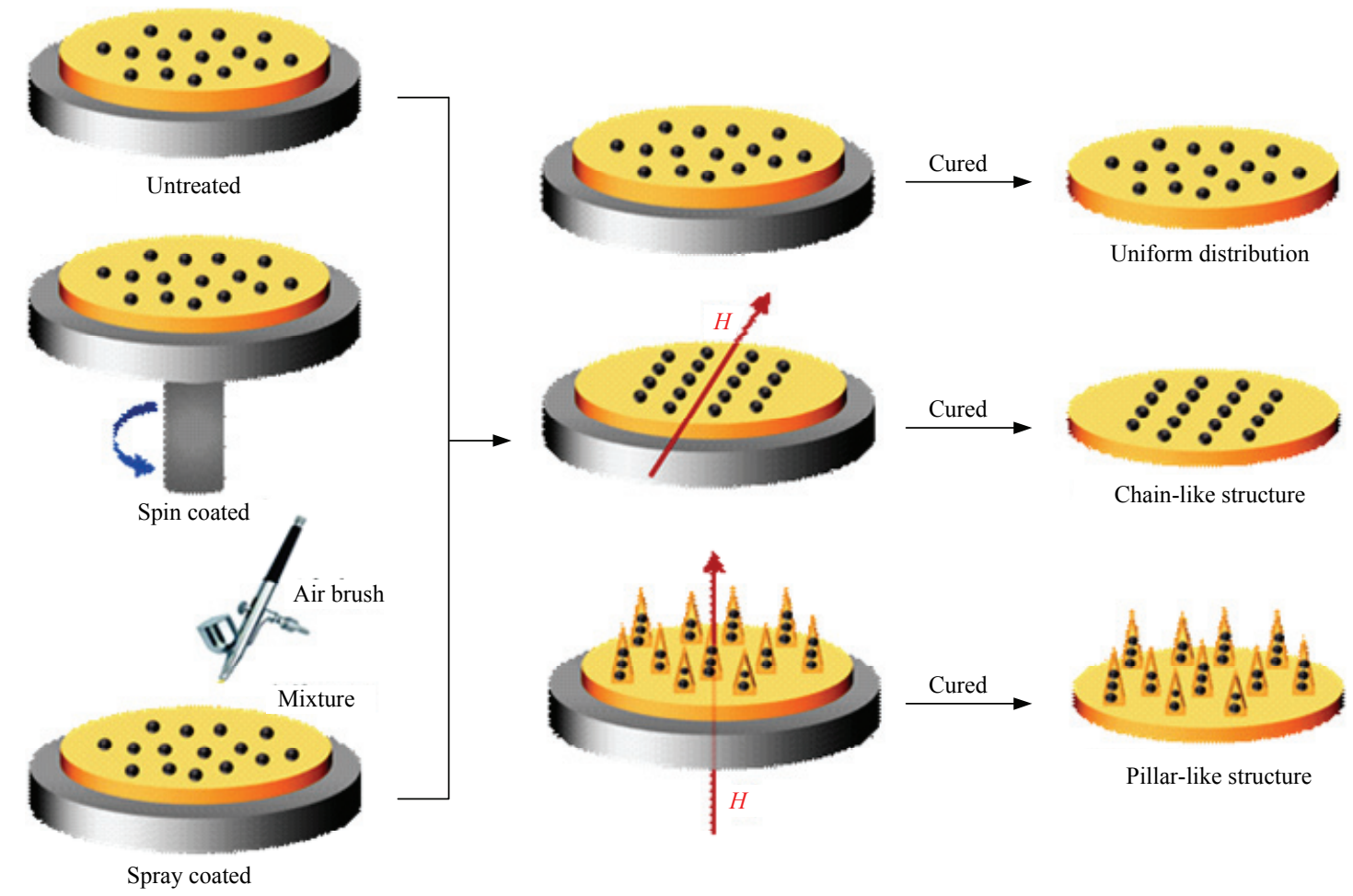

Fig. 2 The fabrication of magnetoresponsive composite elastomers via spin coating, spray coating, and magnetic particle-assisted $\operatorname{process}^{[78]}$.

field-assisted process, anisotropic magnetoresponsive composite elastomers are produced with ease (Fig. 2). The three-dimensional (3D) printing technique as an intelligent manufacturing technology is generally adopted when the specimens require subtle internal structures or intricate geometries, while the traditional preparation methods fail to satisfy these requirements.

\subsection{Spin and spray coating}

The spin coating as a facile fabrication method is usually used to fabricate magnetoresponsive films. Whilst fabricating ${ }^{[73-75]}$, the mixture containing elastomer matrix and magnetic particles needs to be prepared at first. After spin coating on a spin coater and curing, the magnetoresponsive films are fabricated. The thickness of films can be controlled by regulating the rotational speed and time. The spray coating used to fabricate magnetoresponsive micropillars is different from the spin coating in which the prepared mixture not only contains ingredients but also admixes an organic solvent to form a solution and the instrument used is the airbrush.
Generally, the uncured mixture whether it is not treated or spin coated or spray coated, can spontaneously form motile magnetoresponsive micropillars along the axial direction in the presence of an external magnetic field $^{[76,77]}$. And the parameters related to spin or spray coating and magnetic particle-assisted molding process directly determine the morphology of magnetoresponsive micropillars.

Yang et al. ${ }^{[78]}$ investigated how these parameters including the mixture ratio of components, the amount of spray solution, the spray distance between airbrush and substrate, and the intensity of external magnetic field affected the size and density of magnetoresponsive pillars. With the increase of magnetic particles' concentration in the mixture, the diameter and height of micropillars would increase. Conversely, the increase of elastomer matrices or organic solvent would result in a decrease in the size of pillars. A small amount of spray solution, farther airbrush-substrate distance, and weak external magnetic field led to the generation of small diameter and pillar height. Rather, when increasing the amount of spray solution, shortening the 

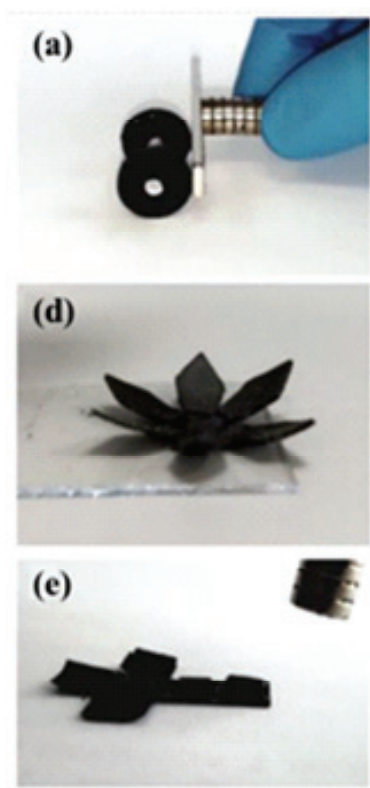

(b)
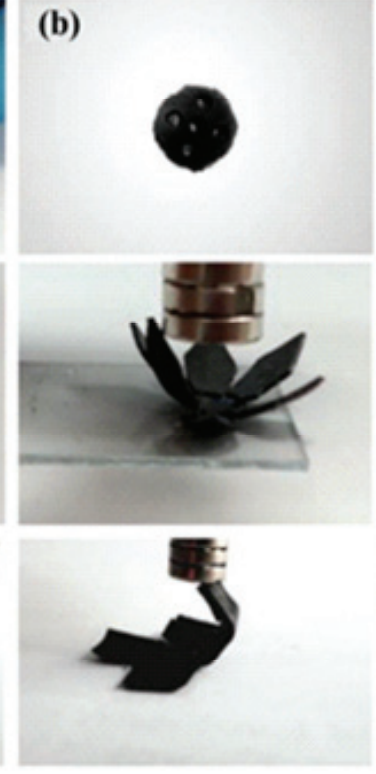

(c)
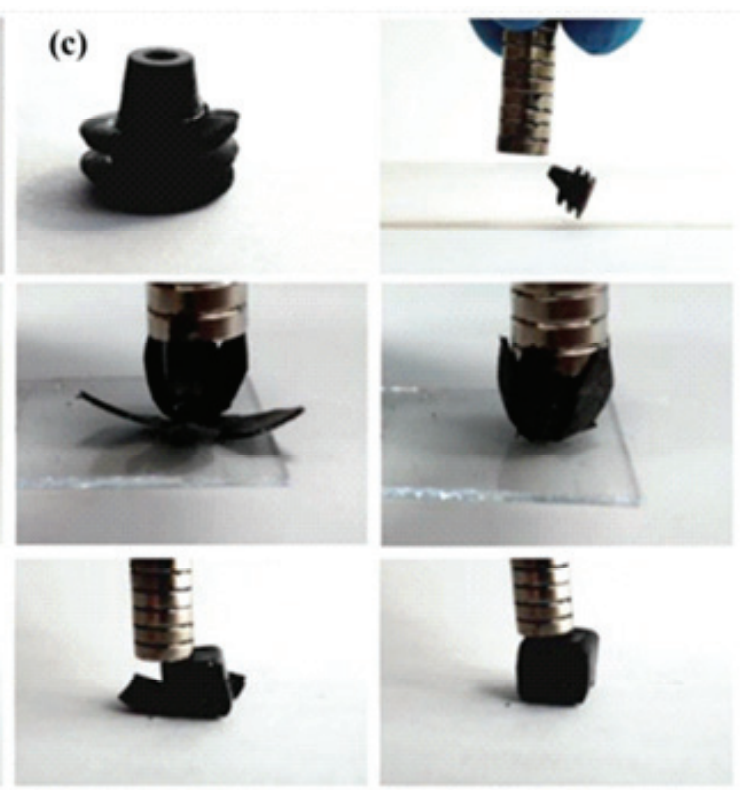

Fig. 3 The 3D printed magnetic objects obtained through digital light processing. (a) Printed two wheels; (b) printed sphere; (c) printed cone-like structure; (d) printed flower which was enclosed upon a magnetic field; (e) 2D structure could be able to create a 3D cube when exposed to a magnetic field ${ }^{[86]}$.

airbrush-substrate distance, enhancing the applied magnetic field, larger diameter and micropillar height would generate.

\subsection{D printing}

$3 \mathrm{D}$ printing ${ }^{[79]}$ as a versatile additive manufacturing technology is superior to traditional manufacturing due to its distinctive advantages in terms of printability of abundant raw materials, diverse patterns designed by software, reduction of desired materials, templates free, so forth ${ }^{[80]}$. The $3 \mathrm{D}$ printing is the process where composites are continuously deposited layer-by-layer, so that the composites used should be rapidly prototyped during the printing process. Direct Ink Writing (DIW), Fused Filament Fabrication (FFF) and stereolithography (SLA) are three main printing methodologies ${ }^{[81]}$. Among them, DIW is the extruding process of viscous inks through a nozzle, FFF involves the deposition of thermoplastic materials $^{[82]}$, and SLA is based on the photopolymerization of photocurable resins upon irradiation ${ }^{[83]}$. Most importantly, these related printing parameters, such as the characteristics of raw materials, the extrusion pressure, the nozzle diameter, determine the quality of printed magnetoresponsive composite elastomers. Recently studies have demonstrated the capability of magnetically assisted 3D printing technology to fabricate 3D magnetoresponsive composite elastomers with complex shapes ${ }^{[84,85]}$.

Kim et al. ${ }^{[19]}$ carried out a magnetically assisted 3D printing approach based on DIW to print various planar and 3D soft structures, whose shapes could be untethered fast transferred under an external magnetic actuation. The magnetic composite ink they prepared was composed of silicone rubber and $\mathrm{NdFeB}$ magnetizable microparticles. Lantean et al. ${ }^{[86]}$ printed the optimized formulations, where urethane-acrylate resins, $\mathrm{Fe}_{3} \mathrm{O}_{4}$ nanoparticles, and butyl acrylate were used to tune viscosity as the diluent, into magnetic responsive samples using digital light processing. These 3D-printed magnetic samples have spring, wheel, sphere, flower, cone and planar structure, whose movements could be controlled by an external magnetic field (Fig. 3). Unlike the mixing of elastomer with magnetic particles, Bastola et $a l .{ }^{[87]}$ attained sandwich structured magnetoresponsive elastomers by preparing two printing cartridges respectively filled with magnetic suspension and elastomer resin. They extruded and UV light cured elastomer resin as a bottom layer firstly, then deposited magnetic suspension on top of resin, and finally dispensed elastomer resin once again to encapsulate the magnetic 


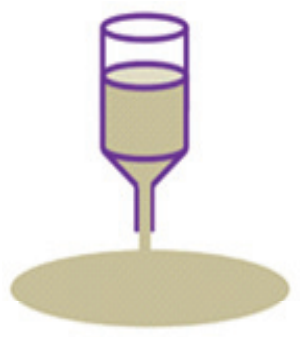

(1)

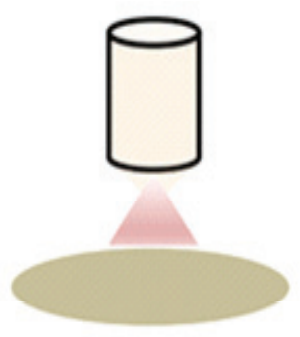

(2)

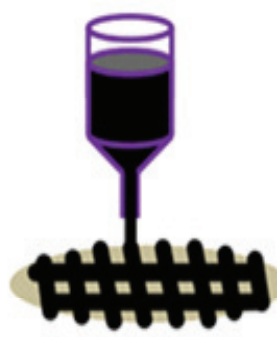

(3)

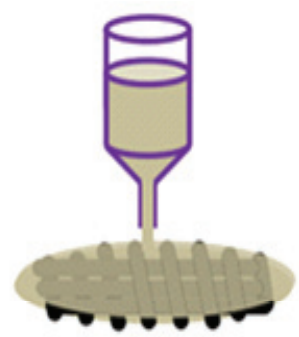

(4)

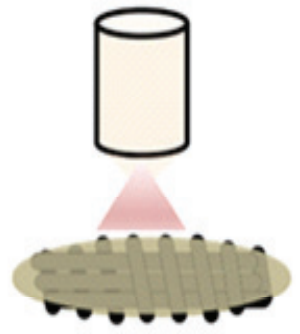

(5)

(1) Printing of elastomer matrix

Elastomer matrix
UV light
MR fluid

(2) Curing with UV light

(3) Printing of magnetic suspension

(4) Printing of elastomer matrix

(5) Curing with UV light

Fig. 4 Schematic illustration for printing sandwich structured magnetoresponsive elastomers via 3D printing technology ${ }^{[87]}$.

patterns (Fig. 4).

\section{Bionic locomotion applications of magne- toresponsive composite elastomers}

In nature, varieties of living creatures morphing motion modalities flourish, the crawling and rolling of caterpillars $^{[88]}$, the anchor telescoping motion of inch${ }_{\text {worms }}{ }^{[89]}$, the upward swimming of jellyfishes ${ }^{[90]}$, the undulatory topographical motion of batoidea ${ }^{[91]}$, the leaping of nematodes ${ }^{[92]}$, so forth ${ }^{[93-95]}$. These instances have stimulated scientists to build advanced actuators which can not only dynamically change their morphologies, but also motion at diverse environments when exposed to a stimulus. As a class of smart soft materials, magnetoresponsive composite elastomers can change their properties by regulating the external magnetic field direction and strength, especially their shapes can be stretched, warped or recoiled upon a magnetic field ${ }^{[96,97]}$. Of course, the bionic magnetic actuators made of magnetoresponsive composite elastomers are put forward. By manipulating the magnetic field, the bionic locomotion actuators are expected to enable multimodal locomotion, fluid flow adjustment, object clamping, target transporting, and have great potential applications in the field of artificial soft robotics, microfluidic systems, biomedical devices, flexible electronics, etc.

\subsection{Shape-morphing locomotion inspiration}

Biological systems contain plentiful living organisms that exhibit remarkable shape-morphing abilities, more interestingly, they are capable of transforming deformations into diverse motions for better survival in dangerous habitats. Among them, the familiar caterpillars and inchworms show shape-morphing behaviors during locomotion due to their extremely soft bodies, which are the two main imitative organisms of the bionic locomotion actuators. Therefore, to better understand the generation of these bionic devices, it is necessary to briefly illuminate the motion mechanisms of these two typical organisms before the statement of bionic locomotion actuators based on the magnetoresponsive composite elastomers.

Soft-bodied caterpillar ${ }^{[98]}$ crawls forward by the movement of contraction wave from tail to head. As the hump wave moves forward, each segment raises from the ground and passes to the neighbor in front. When its head or thorax suffers shocks, the contraction wave crawls from head to tail and elicits the backward locomotion. All of its legs uplift from the ground, except for the terminal clasper as an anchor to jerk the whole body. As the peristaltic wave arrives at the terminal clasper, all legs reattach the ground and consequently, a whole reverse gallop is completed. Apart from the aforemen- 
tioned two undulating gaits, caterpillar produces special rolling locomotion to quickly escape from danger. However, rolling locomotion no longer depends on the motion of the peristaltic wave. Caterpillar relies on the anchoring of terminal clasper, recoils and transforms into a backward rolling wheel. The resulting escape speed is almost 40 times the normal walking speed.

Inchworm is divided into three different functional parts: the body, the front feet, and the back feet ${ }^{[99]}$. The locomotion of inchworm is similar to the caterpillar, which is an anchor crawling movement based on the contraction and extension of abdominal longitudinal muscles ${ }^{[100]}$. In each crawling cycle, firstly, inchworm anchors on the front feet and bends the body through the contraction of abdominal longitudinal muscles so that the back feet crawl forward. Subsequently, inchworm changes its anchors from the front feet to the rear feet and extends the body as the stretch of abdominal longitudinal muscles, which causes the front feet to crawl forward ${ }^{[101]}$. And inchworm nearly supports most of its body all the time ${ }^{[102]}$.

\subsection{Bionic locomotion actuators}

Getting a big source of inspiration from the shape-shifting behaviors and the resulting motions of creatures, scientists have made energetic efforts to develop bionic actuators based on magnetoresponsive composite elastomers that combine the elasticity of elastomer matrices with the non-contacting magnetic actuation. Such actuators can deform in response to the external magnetic field, thereby execute specific tasks, creature-like locomotion in different environments, obstacles crossing, target gripping and transporting, providing perspectives on developing artificial soft robotics and biomedical devices.

$\mathrm{Hu}$ et al. ${ }^{[103]}$ embedded hard NdFeB microparticles into silicone elastomer to devise a rectangular shaped, isotropic, magnetoresponsive soft actuator whose shape could be varied upon a magnetic field. Moreover, theoretical and experimental discussions demonstrated that the bionic actuator could imitate the locomotion modes of some organisms and even reversibly switch between different locomotion modes under the control of a magnetic field. Specifically, this bionic actuator crawled on the ground or the liquid surface slowly and rolled over the ground or directionally from the ground onto the liquid surface rapidly like a caterpillar. It moved like an inchworm and the variation of its curvature would tune its stride length. Besides, it was capable of gathering momentum by bumping the substrate to leap upward like nematodes and overcoming gravity to swim upward underwater like jellyfishes.

Recently, Ren et al. ${ }^{[104]}$ designed a jellyfish soft actuator that was completely different from the rectangular-shaped actuator just introduced, it was more similar to jellyfish in appearance and swimming behavior. This jellyfish soft actuator was characterized by a magnetoresponsive composite elastomer core and eight lappets as illustrated in Fig. 5a. The magnetoresponsive composite elastomer was composed of $\mathrm{NdFeB}$ microparticles and the silicone rubber with a mass ratio of 1:1. And the measurement of the static contact angle between water and the magnetoresponsive composite elastomer demonstrated that the composite material was hydrophobic. When swimming, the magnetoresponsive composite elastomer core would beat up and down eight lappets of this actuator by changing the external magnetic field (Fig. 5b).

The bionic actuators made of anisotropic magnetoresponsive elastomers where magnetic particles are aligned horizontally or vertically, have also been reported. Kim et al.${ }^{[105]}$ proposed a creative design concept combining the self-assembly of magnetic particles and rapid photopolymerization and devised anisotropic actuators composing multiple magnetically responsive parts where magnetic particles aligned in different orientations, so that each part could move in diverse angles under a homogeneous magnetic field. To confirm the bionic locomotion feasibility of the anisotropic actuators, the authors fabricated two bionic actuators by dispersing superparamagnetic colloidal nanocrystal clusters into the poly(ethylene glycol) diacrylate photocurable resin. The former consisted of two inner parts with parallel magnetic axes and two outer parts with vertical magnetic axes and the latter contained four parts with different magnetic axes. By carefully modulating the magnetic field direction, the former actuator crept like an inchworm and the latter slithered like a snake.

Inspired by flexible legs of polypod animals, Lu et al. ${ }^{[106]}$ presented a thin sheet actuator integrated 
(a)

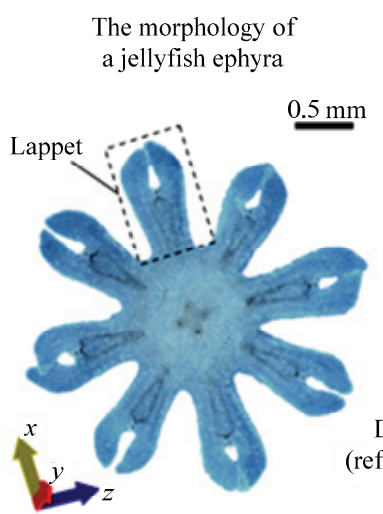

(b)

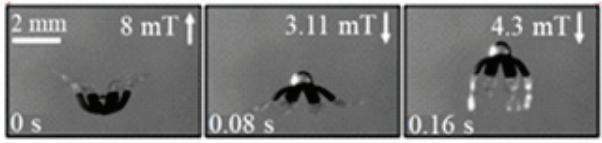

The schematic of the jellyfish robot design

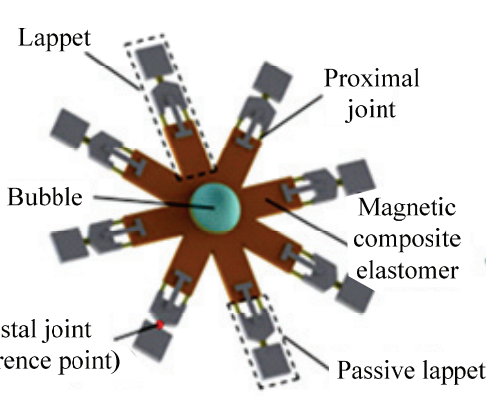

The photograph of the jellyfish robot

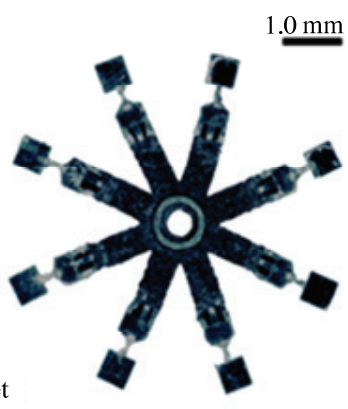

Fig. 5 Design and swimming behavior of the jellyfish-inspired swimming soft actuator ${ }^{[104]}$. (a) Imitating the morphology of a jellyfish ephyra, the swimming soft actuator had a magnetoresponsive composite elastomer core and eight lappets; (b) the swimming soft actuator beat up and down its lappets under a magnetic actuation.

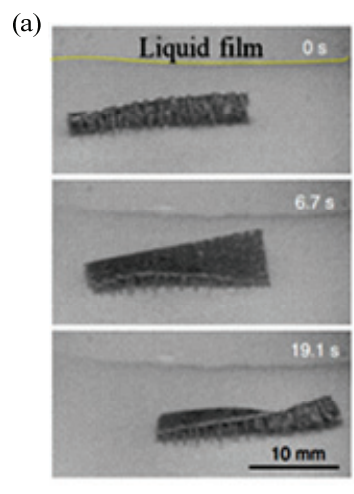

(d)

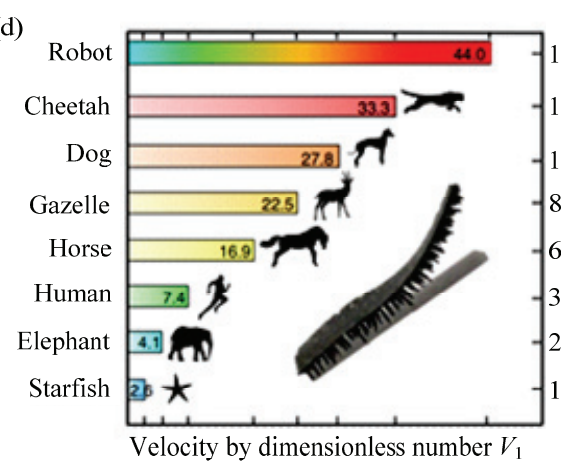

(b)
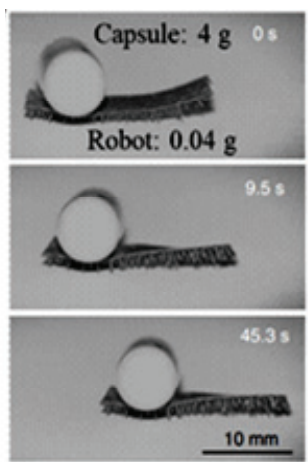

(e) (c)

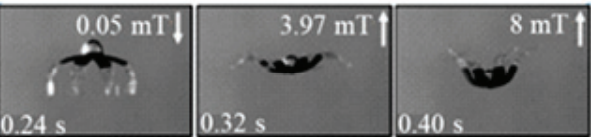

\section{(c)}
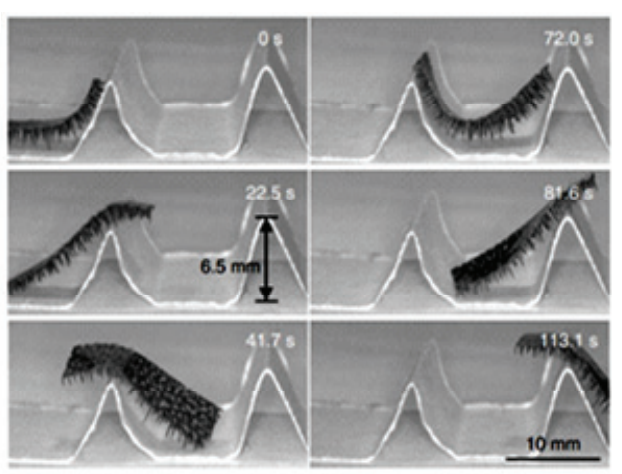

(e)

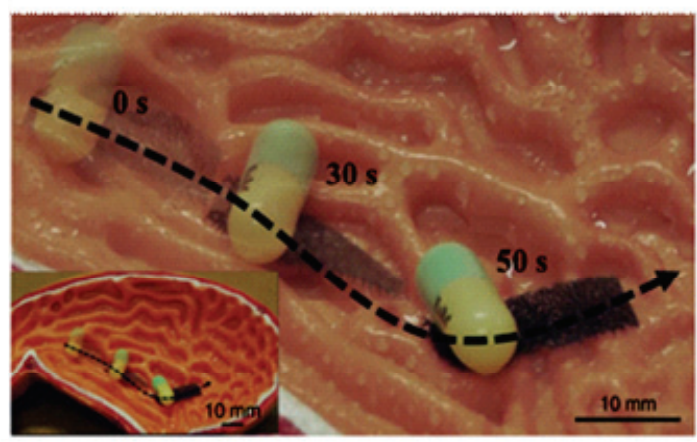

Fig. 6 (a) The locomotion on a wet surface with a liquid film; (b) the locomotion with a loading 100 times of its weight; (c) climbing steep obstacles 10 times higher than its leg; (d) comparison of the locomotion speed between the multilegged actuator and other animals; (e) the drug transport on a human stomach model ${ }^{[106]}$.

with multiple soft tapered feet where magnetic particles were aligned vertically. This bionic actuator was made of polydimethylsiloxane and iron microparticles by a magnetic particle-assisted molding process, which performed excellent deformability, locomotion ability, carrying capability and crossing obstacle capacity. The Young modulus of this bionic actuator was $2 \mathrm{MPa}$. It could walk on a wet surface (Fig. 6a), move on a rigid substrate carrying a cargo 100 times heavier than its weight (Fig. 6b), even climb the steep obstacles that 
(a)

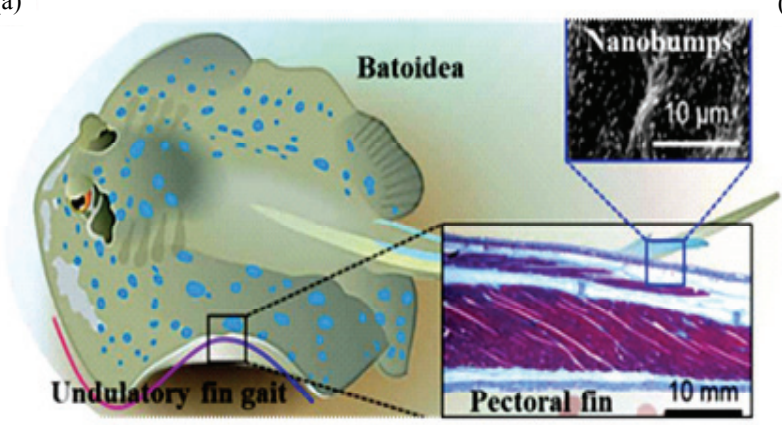

(c)

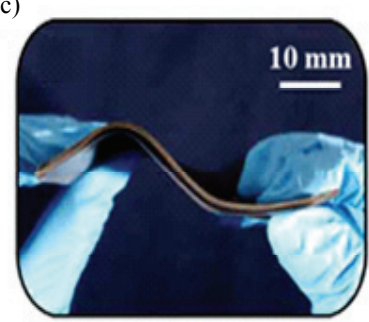

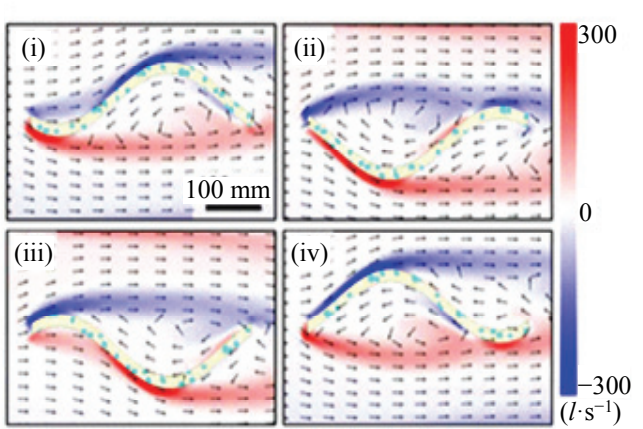

(d)

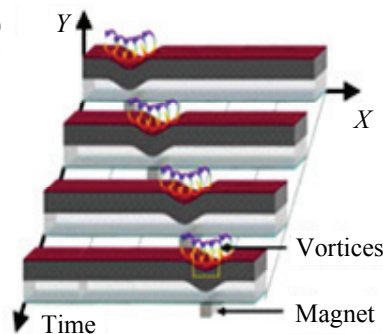

Fig. 7 Design and the dynamic antifouling behavior of the batoidea-inspired magnetoresponsive multilayered composite ${ }^{[91]}$. (a) The conceptual illustration of the undulatory gait of a batoidea and the histological cross-section of the petrol fin; (b) the schematic illustrations of vortices induced by the dynamic undulatory topographical locomotion of a batoidea; (c) the image and the cross-sectional stereo zoom microscope image of the multilayered composite; (d) the schematic illustration of the undulatory topographical wave.

were 10 times higher than its leg continuously (Fig. 6c), and reach an ultrafast locomotion speed of $40 \mathrm{limb}$ length/s, which was quicker than those of humans and cheetah (Fig. 6d). Notably, it was capable of carrying a medical tablet that weighed twice as much as its own weight to locomotion on an artificial human stomach with complex internal structure and wet surface which demonstrated its potential applications in the biomedical field (Fig. 6e).

The dynamic undulatory topographical locomotion of a batoidea (Figs. 7a and 7b) inspired Ko et al. ${ }^{[91]}$ to propose an efficient and sustainable surface antifouling strategy that utilized the undulatory topographical waves induced by the dynamic shape-morphing locomotion of a class of new antifouling materials to clear away foulants and restrain the formation of biofilms without any surface modification (Fig. 7d). This new antifouling material was the magnetoresponsive multilayered composite where the magnetic artificial muscle layer was prepared by dispersing carbonyl irons into PDMS dominated (Fig. 7c). The antifouling experiments showed that with the aid of the reciprocating motion of a magnet, the undulatory topographical waves generated by the deformation of the composite eventually resulted in the directional fluid flow, thereby cleared Escherichia coli on the surface of the composite and prevented the formation of biofilms.

\section{Summary and outlook}

The miraculous ecosystem provides endless inspirations for technological development ${ }^{[107-110]}$. Inspired by the shape-morphing locomotion of living organisms, the bionic actuators based on magnetoresponsive composite elastomers which can deform and motion in response to the external magnetic field have been developed. The development and application of bionic locomotion systems is a burgeoning area especially in the field of artificial soft robots, microfluidic systems, biomedicine, etc.

Despite the progress that has been achieved, there are still some challenges and difficulties in the development of bionic locomotion actuators based on magnetoresponsive composite elastomers. Firstly, it is very difficult to apply more complex biological principles into innovative design strategies for creating locomotion actuators in applications. Secondly, there remains a certain disparity between the bionic locomotion actuator, and the natural living organisms in rapid morphing, 
steerability, and multimodal locomotion conversion. Most bionic locomotion actuators can imitate a single locomotion mode of a creature, but it is difficult to imitate diverse locomotion modes of multiple creatures, and convert quickly and reversibly multimodal locomotion. Thirdly, the magnetic field is selected to be an external stimulus due to its battery-free remote control, directional adjustment, ease of use even outside the lab-context, and the ability to penetrate most objects simply and harmlessly, etc. However, as for the controllability of the bionic locomotion actuators based on magnetoresponsive composite elastomers by a magnetic field, in most cases, the entire actuator is regulated as a whole by the applied magnetic field, but the precise regulation of each small part of actuators by the magnetic stimulus has not been implemented. In conclusion, there is still huge room for the future exploration of bionic locomotion actuators based on magnetoresponsive composite elastomers.

\section{Acknowledgment}

This work was supported by the National Key R\&D Program of China (Grant No. 2018YFA0209500), the National Natural Science Foundation of China (Grant Nos. 21673197, 21621091, 21808191, and 21975209), the 111 Project (Grant No. B16029), the Fundamental Research Funds for the Central Universities of China (Grant No. 20720190037), the Natural Science Foundation of Fujian Province of China (Grant No. 2018J06003), and CAS Key Laboratory of Bio-inspired Materials and Interfacial Science, Technical Institute of Physics and Chemistry, Chinese Academy of Sciences.

Open Access This article is licensed under a Creative Commons Attribution 4.0 International License, which permits use, sharing, adaptation, distribution and reproduction in any medium or format, as long as you give appropriate credit to the original author(s) and the source, provide a link to the Creative Commons licence, and indicate if changes were made.

The images or other third party material in this article are included in the article's Creative Commons licence, unless indicated otherwise in a credit line to the material. If material is not included in the article's Creative Commons licence and your intended use is not permitted by statutory regulation or exceeds the per- mitted use, you will need to obtain permission directly from the copyright holder.

To view a copy of this licence, visit http://creativecommons.org/licenses/by/4.0/.

\section{References}

[1] Ishige M, Umedachi T, Taniguchi T, Kawahara Y. Exploring behaviors of caterpillar-like soft robots with a central pattern generator-based controller and reinforcement learning. Soft Robotics, 2019, 6, 579-594.

[2] Chiang Y H, Tsai S L, Tee S R, Nair O L, Loh I Y, Liu M H, Wang Z S. Inchworm bipedal nanowalker. Nanoscale, 2018, 10, 9199-9211.

[3] Park S G, Chang C B, Huang W X, Sung H J. Simulation of swimming oblate jellyfish with a paddling-based locomotion. Journal of Fluid Mechanics, 2014, 748, 731-755.

[4] Reed E M, Wallace H R. Leaping locomotion by an insect-parasitic nematode. Nature, 1965, 206, 210-211.

[5] Cao Y T, Liu Y L, Chen Y L, Zhu L L, Yan Y, Chen X. A novel slithering locomotion mechanism for a snake-like soft robot. Journal of the Mechanics and Physics of Solids, 2017, 99, 304-320.

[6] Huffard C L, Boneka F, Full R J. Underwater bipedal locomotion by octopuses in disguise. Science, 2005, 307, 1927.

[7] Gao W, Wang L, Wang X, Liu H. Magnetic driving flowerlike soft platform: Biomimetic fabrication and external regulation. ACS Applied Materials \& Interfaces, 2016, 8, 14182-14189.

[8] Ze Q J, Kuang X, Wu S, Wong J, Montgomery S M, Zhang R D, Kovitz J M, Yang F Y, Qi H J, Zhao R K. Magnetic shape memory polymers with integrated multifunctional shape manipulation. Advanced Materials, 2019, 32, 1906657.

[9] Bi C H, Guix M, Johnson B V, Jing W M, Cappelleri D J. Design of microscale magnetic tumbling robots for locomotion in multiple environments and complex terrains. Micromachines, 2018, 9, 68.

[10] Lee W, Nam J, Kim J, Jung E, Jang G. Effective locomotion and precise unclogging motion of an untethered flexible-legged magnetic robot for vascular diseases. IEEE Transactions on Industrial Electronics, 2018, 65, 1388-1397.

[11] Munoz F, Alici G, Zhou H, Li W, Sitti M. Analysis of magnetic interaction in remotely controlled magnetic devices and its application to a capsule robot for drug delivery. IEEE/ASME Transactions on Mechatronics, 2018, 23, 
298-310.

[12] Gao J, Yan G, Shi Y, Cao H, Huang K, Liu J. Optimization design of extensor for improving locomotion efficiency of inchworm-like capsule robot. Science China Technological Sciences, 2019, 62, 1930-1938.

[13] Halder A, Sun Y. Biocompatible propulsion for biomedical micro/nano robotics. Biosensors and Bioelectronics, 2019, 139, 111334.

[14] Kwok S W, Morin S A, Mosadegh B, So J H, Shepherd R F, Martinez R V, Smith B, Simeone F C, Stokes A A, Whitesides G M. Magnetic assembly of soft robots with hard components. Advanced Functional Materials, 2014, 24, 2180-2187.

[15] Zou J, Lin Y, Ji C, Yang H. A reconfigurable omnidirectional soft robot based on caterpillar locomotion. Soft Robotics, 2018, 5, 164-174.

[16] Joyee E B, Pan Y. A fully three-dimensional printed inchworm-inspired soft robot with magnetic actuation. Soft Robotics, 2019, 6, 333-345.

[17] Ye Z, Diller E, Sitti M. Micro-manipulation using rotational fluid flows induced by remote magnetic micro-manipulators. Journal of Applied Physics, 2012, 112, 064912.

[18] Tang S Y, Zhang X C, Sun S S, Yuan D, Zhao Q B, Yan S, Deng L, Yun G L, Zhang J, Zhang S W, Li W H. Versatile microfluidic platforms enabled by novel magnetorheological elastomer microactuators. Advanced Functional Materials, 2018, 28, 1705484.

[19] Kim Y, Yuk H, Zhao R, Chester S A, Zhao X. Printing ferromagnetic domains for untethered fast-transforming soft materials. Nature, 2018, 558, 274-279.

[20] Chen L, Gong X L, Jiang W Q, Yao J J, Deng H X, Li W H. Investigation on magnetorheological elastomers based on natural rubber. Journal of Materials Science, 2007, 42, 5483-5489.

[21] Jung H S, Kwon S H, Choi H J, Jung J H, Kim Y G. Magnetic carbonyl iron/natural rubber composite elastomer and its magnetorheology. Composite Structures, 2016, 136, 106-112.

[22] Yunus N A, Mazlan S A, Ubaidillah, Aziz S A A, Shilan S T, Wahab N A A. Thermal stability and rheological properties of epoxidized natural rubber-based magnetorheological elastomer. International Journal of Molecular Sciences, 2019, 20, 746.

[23] Puente-Córdova J G, Reyes-Melo M E, Palacios-Pineda L M, Martínez-Perales I A, Martínez-Romero O, Elías-Zúñiga A. Fabrication and characterization of isotropic and anisotropic magnetorheological elastomers, based on silicone rubber and carbonyl iron microparticles. Polymers, 2018, 10, 1343.

[24] Perales-Martínez I A, Palacios-Pineda L M, LozanoSánchez A M, Martínez-Romero O, Puente-Cordova J G, Elías-Zúñiga A. Enhancement of a magnetorheological PDMS elastomer with carbonyl iron particles. Polymer Testing, 2017, 57, 78-86.

[25] Lian C, Lee K H, Lee C H. Effect of temperature and relative humidity on friction and wear properties of silicone-based magnetorheological elastomer. Tribology Transactions, 2017, 61, 238-246.

[26] Ju B, Tang R, Zhang D, Yang B, Yu M, Liao C, Yuan X, Zhang L, Liu J. Dynamic mechanical properties of magnetorheological elastomers based on polyurethane matrix. Polymer Composites, 2016, 37, 1587-1595.

[27] Lian C L, Lee K H, Lee C H. Friction and wear characteristics of magneto-rheological elastomers based on silicone/polyurethane hybrid. Journal of Tribology, 2015, 137, 031607.

[28] Yu M, Qi S, Fu J, Yang P A, Zhu M. Preparation and characterization of a novel magnetorheological elastomer based on polyurethane/epoxy resin IPNs matrix. Smart Materials and Structures, 2015, 24, 045009.

[29] Bica I, Anitas E M, Averis L M E, Kwon S H, Choi H J. Magnetostrictive and viscoelastic characteristics of polyurethane-based magnetorheological elastomer. Journal of Industrial and Engineering Chemistry, 2019, 73, 128-133.

[30] Sun T L, Gong X L, Jiang W Q, Li J F, Xu Z B, Li W H. Study on the damping properties of magnetorheological elastomers based on cis-polybutadiene rubber. Polymer Testing, 2008, 27, 520-526.

[31] Al-Milaji K N, Hadimani R L, Gupta S, Pecharsky V K, Zhao H. Inkjet printing of magnetic particles toward anisotropic magnetic properties. Science Reports, 2019, 9, 16261.

[32] Sun J Z, Bao B, He M, Zhou H H, Song Y L. Recent advances in controlling the depositing morphologies of inkjet droplets. ACS Applied Materials \& Interfaces, 2015, 7, 28086-28099.

[33] Ben S, Zhou T T, Ma H, Yao J J, Ning Y Z, Tian D L, Liu K S, Jiang L. Multifunctional magnetocontrollable superwettable-microcilia surface for directional droplet manipulation. Advanced Science, 2019, 6, 1900834.

[34] Alhnan M A, Okwuosa T C, Sadia M, Wan K W, Ahmed W, Arafat B. Emergence of 3D printed dosage forms: Opportunities and challenges. Pharmaceutical Research, 2016, 33, 1817-1832.

[35] Rahimi A, Mashak A. Review on rubbers in medicine: Natural, silicone and polyurethane rubbers. Plastics, Rubber 
and Composites, 2013, 42, 223-230.

[36] Wang Y L, Hu Y, Wang Y L, Deng H X, Gong X L, Zhang P Q, Jiang W Q, Chen Z Y. Magnetorheological elastomers based on isobutylene-isoprene rubber. Polymer Engineering \& Science, 2006, 46, 264-268.

[37] Xu Z D, Suo S, Zhu J T, Guo Y Q. Performance tests and modeling on high damping magnetorheological elastomers based on bromobutyl rubber. Journal of Intelligent Material Systems and Structures, 2017, 29, 1025-1037.

[38] Zhang W, Gong X L, Jiang W Q, Fan Y C. Investigation of the durability of anisotropic magnetorheological elastomers based on mixed rubber. Smart Materials and Structures, 2010, 19, 085008.

[39] Wang Y H, Zhang X R, Oh J, Chung K. Fabrication and properties of magnetorheological elastomers based on CR/ENR self-crosslinking blends. Smart Materials and Structures, 2015, 24, 095006.

[40] Qi S, Yu M, Fu J, Zhu M, Xie Y P, Li W. An EPDM/MVQ polymer blend based magnetorheological elastomer with good thermostability and mechanical performance. Soft Matter, 2018, 14, 8521-8528.

[41] Kim Y H, Ahn W J, Choi H J, Seo Y. Fabrication and magnetic stimuli-response of polydopamine-coated core-shell structured carbonyl iron microspheres. Colloid and Polymer Science, 2015, 294, 329-337.

[42] Kwon S H, An J S, Choi S Y, Chung K H, Choi H J. Poly(glycidyl methacrylate) coated soft-magnetic carbonyl iron/silicone rubber composite elastomer and its magnetorheology. Macromolecular Research, 2019, 27, 448-453.

[43] Schrödner M, Pflug G. Magnetomechanical properties of composites and fibers made from thermoplastic elastomers (TPE) and carbonyl iron powder (CIP). Journal of Magnetism and Magnetic Materials, 2018, 454, 258-263.

[44] Kwon S H, Lee C J, Choi H J, Chung K H, Jung J H. Viscoelastic and mechanical behaviors of magneto-rheological carbonyl iron/natural rubber composites with magnetic iron oxide nanoparticle. Smart Materials and Structures, 2019, 28, 045012 .

[45] Chae H S, Kim S D, Piao S H, Choi H J. Core-shell structured $\mathrm{Fe}_{3} \mathrm{O}_{4} @ \mathrm{SiO}_{2}$ nanoparticles fabricated by sol-gel method and their magnetorheology. Colloid and Polymer Science, 2016, 294, 647-655.

[46] Jung H S, Choi H J. Hydrothermal fabrication of octahedral-shaped $\mathrm{Fe}_{3} \mathrm{O}_{4}$ nanoparticles and their magnetorheological response. Journal of Applied Physics, 2015, 117, 17E708.

[47] Ruan X H, Pei L, Xuan S H, Yan Q F, Gong X L. The rheological responds of the superparamagnetic fluid based on $\mathrm{Fe}_{3} \mathrm{O}_{4}$ hollow nanospheres. Journal of Magnetism and Magnetic Materials, 2017, 429, 1-10.

[48] Mordina B, Tiwari R K, Setua D K, Sharma A. Magnetorheology of polydimethylsiloxane elastomer $/ \mathrm{FeCo}_{3}$ nanocomposite. The Journal of Physical Chemistry C, 2014, 118, 25684-25703.

[49] Soledad Antonel P, Jorge G, Perez O E, Butera A, Gabriela Leyva A, Martin Negri R. Magnetic and elastic properties of $\mathrm{CoFe}_{2} \mathrm{O}_{4}$-polydimethylsiloxane magnetically oriented elastomer nanocomposites. Journal of Applied Physics, 2011, 110, 043920.

[50] Vasudevan M P, Sudeep P M, Al-Omari I A, Kurian P, Ajayan P M, Narayanan T N, Anantharaman M R. Enhanced microactuation with magnetic field curing of magnetorheological elastomers based on iron-natural rubber nanocomposites. Bulletin of Materials Science, 2014, 38, 689-694.

[51] Kumar V, Lee D J. Mechanical properties and magnetic effect of new magneto-rheological elastomers filled with multi-wall carbon nanotubes and iron particles. Journal of Magnetism and Magnetic Materials, 2019, 482, 329-335.

[52] Xia Z Z, Wu X X, Peng G R, Wang L, Li W H, Wen W J. A novel nickel nanowire based magnetorheological material. Smart Materials and Structures, 2017, 26, 054006.

[53] Hapipi N, Aziz S A A, Mazlan S A, Ubaidillah, Choi S B, Mohamad N, Khairi M H A, Fatah A Y A. The field-dependent rheological properties of plate-like carbonyl iron particle-based magnetorheological elastomers. Results in Physics, 2019, 12, 2146-2154.

[54] Sorokin V V, Stepanov G V, Shamonin M, Monkman G J, Khokhlov A R, Kramarenko E Y. Hysteresis of the viscoelastic properties and the normal force in magnetically and mechanically soft magnetoactive elastomers: Effects of filler composition, strain amplitude and magnetic field. Polymer, 2015, 76, 191-202.

[55] Winger J, Schümann M, Kupka A, Odenbach S. Influence of the particle size on the magnetorheological effect of magnetorheological elastomers. Journal of Magnetism and Magnetic Materials, 2019, 481, 176-182.

[56] Cvek M, Mrlik M, Ilcikova M, Plachy T, Sedlacik M, Mosnacek J, Pavlinek V. A facile controllable coating of carbonyl iron particles with poly(glycidyl methacrylate): A tool for adjusting MR response and stability properties. Journal of Materials Chemistry C, 2015, 3, 4646-4656.

[57] Cvek M, Mrlík M, Ilčíková M, Mosnáček J, Münster L, Pavlínek V. Synthesis of silicone elastomers containing silyl-based polymer-grafted carbonyl iron particles: An ef- 
ficient way to improve magnetorheological, damping, and sensing performances. Macromolecules, 2017, 50, 2189-2200

[58] Cvek M, Mrlik M, Sevcik J, Sedlacik M. Tailoring performance, damping, and surface properties of magnetorheological elastomers via particle-grafting technology. Polymers, 2018, 10, 1411.

[59] Rabindranath R, Böse H. On the mobility of iron particles embedded in elastomeric silicone matrix. Journal of Physics: Conference Series, 2013, 412, 012034.

[60] Wang G S, Ma Y Y, Tong Y, Dong X F. Synthesis, characterization and magnetorheological study of 3-aminopropyltriethoxysilane-modified $\mathrm{Fe}_{3} \mathrm{O}_{4}$ nanoparticles. Smart Materials and Structures, 2016, 25, 035028.

[61] Khairi M H A, Mazlan S A, Ubaidillah, Ahmad K Z K, Choi S B, Aziz S A A, Yunus N A. The field-dependent complex modulus of magnetorheological elastomers consisting of sucrose acetate isobutyrate ester. Journal of Intelligent Material Systems and Structures, 2017, 28, 1993-2004.

[62] Tian T F, Nakano M. Fabrication and characterisation of anisotropic magnetorheological elastomer with $45^{\circ}$ iron particle alignment at various silicone oil concentrations. Journal of Intelligent Material Systems and Structures, 2017, 29, 151-159.

[63] Kimura Y, Kanauchi S, Kawai M, Mitsumata T, Tamesue S, Yamauchi T. Effect of plasticizer on the magnetoelastic behavior for magnetic polyurethane elastomers. Chemistry Letters, 2015, 44, 177-178.

[64] Nayak B, Dwivedy S K, Murthy K S. Fabrication and characterization of magnetorheological elastomer with carbon black. Journal of Intelligent Material Systems and Structures, 2014, 26, 830-839.

[65] Poojary U R, Hegde S, Gangadharan K V. Experimental investigation on the effect of carbon nanotube additive on the field-induced viscoelastic properties of magnetorheological elastomer. Journal of Materials Science, 2017, 53, 4229-4241.

[66] Chen S P, Chen J, Xu K, Pan R K, Peng Z, Ma L, Wu S C. Effect of coal gangue/carbon black/multiwall carbon nanotubes hybrid fillers on the properties of natural rubber composites. Polymer Composites, 2016, 37, 3083-3092.

[67] Fan L L, Wang G P, Wang W J, Lu H L, Yang F F, Rui X T. Size effect of carbon black on the structure and mechanical properties of magnetorheological elastomers. Journal of Materials Science, 2018, 54, 1326-1340.

[68] Lu H L, Wang W J, Yang F F, Wang G P, Rui X T. Effect of carbon black with large particle size on dynamic mechanical analysis of magnetorheological elastomers (MREs). Materials Research Express, 2018, 5, 095703.

[69] Aziz S A A, Mazlan S A, Ismail N I N, Ubaidillah U, Choi S B, Khairi M H A, Yunus N A. Effects of multiwall carbon nanotubes on viscoelastic properties of magnetorheological elastomers. Smart Materials and Structures, 2016, 25, 077001.

[70] Aziz S A A, Ubaidillah, Mazlan S A, Ismail N I N, Choi S B. Implementation of functionalized multiwall carbon nanotubes on magnetorheological elastomer. Journal of Materials Science, 2018, 53, 10122-10134.

[71] Gong X L, Fan Y C, Xuan S H, Xu Y G, Peng C. Control of the damping properties of magnetorheological elastomers by using polycaprolactone as a temperature-controlling component. Industrial \& Engineering Chemistry Research, 2012, 51, 6395-6403.

[72] Yun G L, Tang S Y, Sun S S, Yuan D, Zhao Q B, Deng L, Yan S, Du H P, Dickey M D, Li W H. Liquid metal-filled magnetorheological elastomer with positive piezoconductivity. Nature Communications, 2019, 10, 1300.

[73] Feng J B, Xuan S H, Lv Z Q, Pei L, Zhang Q C, Gong X L. Magnetic-field-induced deformation analysis of magnetoactive elastomer film by means of DIC, LDV, and FEM. Industrial \& Engineering Chemistry Research, 2018, 57, 3246-3254.

[74] Marchi S, Casu A, Bertora F, Athanassiou A, Fragouli D. Highly magneto-responsive elastomeric films created by a two-step fabrication process. ACS Applied Materials \& Interfaces, 2015, 7, 19112-19118.

[75] Feng J B, Xuan S H, Ding L, Gong X L. Magnetoactive elastomer/PVDF composite film based magnetically controllable actuator with real-time deformation feedback property. Composites Part A: Applied Science and Manufacturing, 2017, 103, 25-34.

[76] Cao M Y, Jin X, Peng Y, Yu C M, Li K, Liu K S, Jiang L. Unidirectional wetting properties on multi-bioinspired magnetocontrollable slippery microcilia. Advanced Materials, 2017, 29, 1606869.

[77] Huang Y, Stogin B B, Sun N, Wang J, Yang S, Wong T S. A switchable cross-species liquid repellent surface. Advanced Materials, 2017, 29, 1604641.

[78] Yang C, Wu L, Li G. Magnetically responsive superhydrophobic surface: In situ reversible switching of water droplet wettability and adhesion for droplet manipulation. ACS Applied Materials \& Interfaces, 2018, 10, 20150-20158.

[79] Hofmann M. 3D printing gets a boost and opportunities with polymer materials. ACS Macro Letters, 2014, 3, 382-386. 
[80] Lu L, Guo P, Pan Y Y. Magnetic-field-assisted projection stereolithography for three-dimensional printing of smart structures. Journal of Manufacturing Science and Engineering, 2017, 139, 071008.

[81] Bekas D G, Hou Y, Liu Y, Panesar A. 3D printing to enable multifunctionality in polymer-based composites: A review. Composites Part B: Engineering, 2019, 179, 107540.

[82] Khatri B, Lappe K, Noetzel D, Pursche K, Hanemann T. A 3D-printable polymer-metal soft-magnetic functional composite-development and characterization. Materials, 2018, 11, 189.

[83] Layani M, Wang X, Magdassi S. Novel materials for 3D printing by photopolymerization. Advanced Materials, 2018, 30, 1706344.

[84] Kokkinis D, Schaffner M, Studart A R. Multimaterial magnetically assisted 3D printing of composite materials. Nature Communications, 2015, 6, 8643.

[85] Schaffner M, Faber J A, Pianegonda L, Rühs P A, Coulter F, Studart A R. 3D printing of robotic soft actuators with programmable bioinspired architectures. Nature Communications, 2018, 9, 878 .

[86] Lantean S, Barrera G, Pirri C F, Tiberto P, Sangermano M, Roppolo I, Rizza G. 3D printing of magnetoresponsive polymeric materials with tunable mechanical and magnetic properties by digital light processing. Advanced Materials Technologies, 2019, 4, 1900505.

[87] Bastola A K, Paudel M, Li L. Development of hybrid magnetorheological elastomers by 3D printing. Polymer, 2018, 149, 213-228.

[88] Vaughan S C, Lin H T, Trimmer B A. Caterpillar climbing: Robust, tension-based omni-directional locomotion. Journal of Insect Science, 2018, 18, 1-8.

[89] Yamamoto T, Konyo M, Tadakuma K, Tadokoro S. High-speed sliding-inchworm motion mechanism with expansion-type pneumatic hollow-shaft actuators for in-pipe inspections. Mechatronics, 2018, 56, 101-114.

[90] Park S G, Kim B, Lee J, Huang W X, Sung H J. Dynamics of prolate jellyfish with a jet-based locomotion. Journal of Fluids and Structures, 2015, 57, 331-343.

[91] Ko H, Park H-H, Byeon H, Kang M, Ryu J, Sung H J, Lee S J, Jeong $\mathrm{H}$ E. Undulatory topographical waves for flow-induced foulant sweeping. Science Advances, 2019, 5, eaax8935.

[92] Backhaus S, Simmonds R W, Loshak A, Davis J C, Packard R E. How and why a parasitic nematode jumps. Nature, 1999, 397, 485-486.

[93] Hu D L, Bush J W. Meniscus-climbing insects. Nature, 2005,
437, 733-736.

[94] Zurek D B, Gorb S N, Voigt D. Locomotion and attachment of leaf beetle larvae Gastrophysa viridula (Coleoptera, Chrysomelidae). Interface Focus, 2015, 5, 20140055.

[95] Behn C, Heinz L, Krüger M. Kinematic and dynamic description of non-standard snake-like locomotion systems. Mechatronics, 2016, 37, 1-11.

[96] Bodelot L, Pössinger T, Danas K, Triantafyllidis N, Bolzmacher C. Magnetorheological elastomers: Experimental and modeling aspects. Mechanics of Composite and Multi-functional Materials, 2016, 7, 251-256.

[97] Bodelot L, Voropaieff J P, Pössinger T. Experimental investigation of the coupled magneto-mechanical response in magnetorheological elastomers. Experimental Mechanics, 2018, 58, 207-221.

[98] Brackenbury J. Caterpillar kinematics. Nature, 1997, 390, 453.

[99] Wang W, Lee J Y, Rodrigue H, Song S H, Chu W S, Ahn S H. Locomotion of inchworm-inspired robot made of smart soft composite (SSC). Bioinspiration \& Biomimetics, 2014, 9, 046006.

[100] Shi Z Y, Pan J, Tian J W, Huang H, Jiang Y R, Zeng S. An inchworm-inspired crawling robot. Journal of Bionic Engineering, 2019, 16, 582-592.

[101] Wang J B, Min J, Fei Y Q, Pang W. Study on nonlinear crawling locomotion of modular differential drive soft robot. Nonlinear Dynamics, 2019, 97, 1107-1123.

[102] Lin H T, Slate D J, Paetsch C R, Dorfmann A L, Trimmer B A. Scaling of caterpillar body properties and its biomechanical implications for the use of a hydrostatic skeleton. The Journal of Experimental Biology, 2011, 214, 1194-1204.

[103] Hu W, Lum G Z, Mastrangeli M, Sitti M. Small-scale soft-bodied robot with multimodal locomotion. Nature, 2018, 554, 81-85.

[104] Ren Z Y, Hu W Q, Dong X G, Sitti M. Multi-functional soft-bodied jellyfish-like swimming. Nature Communications, 2019, 10, 2703.

[105] Kim J, Chung S E, Choi S E, Lee H, Kim J, Kwon S. Programming magnetic anisotropy in polymeric microactuators. Nature Materials, 2011, 10, 747-752.

[106] Lu H J, Zhang M, Yang Y Y, Huang Q, Fukuda T, Wang Z K, Shen Y J. A bioinspired multilegged soft millirobot that functions in both dry and wet conditions. Nature Communications, 2018, 9, 3944.

[107] Zhang J, Zhan K, Wang S L, Hou X. Soft interface design for electrokinetic energy conversion. Soft Matter, 2020, 16, 
2915-2927.

[108] Sheng Z Z, Liu X, Min L L, Wang H L, Liu W, Wang M, Huang L L, Wu F, Hou X. Bioinspired approaches for medical devices. Chinese Chemical Letters, 2017, 28, 1131-1134.

[109] Wu F, Chen S Y, Chen B Y, Wang M, Min L L, Alvarenga J, Ju J, Khademhosseini A, Yao Y, Zhang Y S, Aizenberg J,
Hou X. Bioinspired universal flexible elastomer-based microchannels. Small, 2018, 14, e1702170.

[110] Sheng Z Z, Wang H L, Tang Y L, Wang M, Huang L Z, Min L L, Meng H Q, Chen S Y, Jiang L, Hou X. Tunable liquid gating elastomeric porous system with dynamically controllable gas/liquid transport. Science Advances, 2018, 4, eaao6724. 